\title{
A review: Analysis of fractional order chaotic systems
}

\author{
Niyojeet Shrirao, Alpana Pandey and Ramji Gupta. \\ Department of Electronics and Communication Engineering \\ Maulana Azad National Institute of Technology, Bhopal,462003, India \\ niyojeetshrirao@gmail.com
}

\begin{abstract}
Chaos is generally understood as confusion or disorder. It is the property of systems which are complex andunpredictable in nature.Chaos is often misunderstood as noise, but they are different entities due to the predictable nature of chaos. In this paper different fractional order chaotic systems Fractional Calculus is not a new topic though but the use of it has been applied efficiently in the last few decades. Generally, real objects are fractional order systems, thus fractional order has been implemented on the different chaotic systems which are discussed in the paper and the changes on the systems are studied accordingly. The chaotic system's behavior is observed for fractional order. Each system's chaotic nature is studied for different fractional orders and analyzed to check which order is suitable to observe chaos in the respective chaotic system. Synchronization and applications of the fractional order systems are also discussed.
\end{abstract}

\section{INTRODUCTION}

Chaos was first introduced in 1963 by E.Lorenz[8] Since then chaos has been the center of research for many scientists and research groups around the globe but it has been just a few decades that it's been used efficiently especially in the field of communication. Chaos has played an important role in control applications such as controlling of irregular behavior in devices and systems [5] even in communication privacy with the principle of chaotic masking [17], and in synchronization of two same or different systems which leads to secure communication, chaotic band radio and encryption [5]. Chaotic systems also have applications in short term predictions which can be implemented for predicting the weather, contagious diseases \& economy

A Chaotic system comprises of differential equation where any two solutions at a time are way different for two very similar conditions. Many chaotic systems have been designed to generate chaos for purposes as discussed above. These chaotic systems generate attractors which are nothing but a set of points in phase space, invariant under the dynamics. These attractors are non-periodic in nature hence the conservative systems can't produce them [20].In nonautonomous system chaos may occur for even when there are just 2 variables in the system and in the case of autonomous it can happen with even just three state variables [1]. Most of the times the chaotic systems are of integer order but by studying fractional order calculus it was observed that fractional order chaotic systems are better in the realization of the real objects due to their dynamic nature. The fractional order chaotic systems were found to be more adequate for describing the non-linearsystems in nature as compared to integer order chaotic systems. The fractional order systems are nonlocal in nature since their future state doesn't depend only on their present state but also on the conditions of its past states. [24]

Fractional order calculus is not a new topic. It is almost as old as integer order calculus. In the year 1695, Leibnitz wrote in a reply to de l'Hospital "Thus it follows that $\mathrm{d}^{1 / 2} \mathrm{xwill}$ be equal to $\sqrt{\mathrm{dx}: \mathrm{x}, \ldots}$ from which one-day useful consequences will be drawn". We are familiar with integer order differential and integral operations but what if the order is a non-integer like a fraction or a constant like $\pi$ thus fractional order calculus came into play. There are numerous methods to study fractional calculus, but the best-known method is the Riemann-Liouville which is given by

$$
\frac{d^{\alpha} f(t)}{d t^{\alpha}}=\frac{1}{\Gamma(n-\alpha)} \frac{d^{n}}{d t^{n}} \int_{\alpha}^{t} \frac{f(\tau)}{(t-\tau)^{\alpha-n+1}} d \tau,
$$

for $\mathrm{n}-1 \leq \alpha<\mathrm{n}$, where $\Gamma($.$) is the gamma function [6] [21].$ The main idea is to generalize the differentiation and integration to a fractional(non-integer) order which can be defined by [6]

$$
a D_{t}^{\alpha}=\left\{\begin{array}{cc}
\frac{d^{\alpha}}{d t^{\alpha}}, & R(\alpha)>0 \\
1, & R(\alpha)>0 \\
\int_{a}^{t}(d \tau)^{-\alpha}, & R(\alpha)>0
\end{array}\right.
$$

here $\mathrm{a} D_{t}^{\alpha}$ is the fundamental operator with a and $\mathrm{t}$ as limits, $\alpha$ is the order for operation where $\alpha \in \mathrm{R}$. 
The functions used in the fractional calculus are Beta function, Gamma function and Mittag-Leffler Function. [13]

The basic form of the Gamma function is nothing but the rationalization of the fractional order calculus for all the existing real numbers. The Gamma function can be understood with the help of the following equation. [13] for all z $\in \mathrm{R}$

$$
\Gamma(z)=\int_{0}^{\infty} e^{-u} u^{(z-1)} d u
$$

Beta Function can also be referred to be as Euler integral of the first kind. Beta function's solution can be defined with the Gamma function's help. The following equation represents the Beta integral and its solution in the terms of Gamma Function. [10]

$$
B(r, s)=\int_{0}^{1}(1-v)^{r-1} v^{s-1} d u=\frac{\Gamma(r) \Gamma(s)}{\Gamma(r+s)}=B(s, r)(1.4)
$$

where $r, s \in R$.

Mittag-Leffler Function is utilized prominently in the field of fractional calculus. An analogous role is played by this function in fractional calculus. The equation consists of an exponential function which has its own unique form. The Mittag-Leffler Function's standard equation is as follows [10]

$$
E_{\alpha}(z)=\frac{\sum_{k=0}^{\infty} z^{k}}{\Gamma(\alpha k+1)}(1.5)
$$

Even though the fractional order chaotic systems are more dynamic in nature as compared to integer order they can't be directly implied in time-domain simulations. Thus, various methods were used introduced and are available in the literature, but the Laplace transformation of the Riemann Liouville method is the simplest of all which can be written as

$$
\int_{0}^{\infty} e^{-s t}{ }_{0} D_{t}^{\alpha}=s^{\alpha} L\{f(t)\}-\left.\sum_{k=0}^{n-1}\left(s_{0}^{k}\right) D^{\alpha-k-1} f(t)\right|_{t=0}
$$

for $\mathrm{n}-1 \leq \alpha<\mathrm{n}$, where $\mathrm{s}=\mathrm{j} \omega$ denotes the Laplace variable. If the initial conditions are set to zero, then the formula reduces to

$$
L\left\{\frac{d^{\alpha} f(t)}{d t^{\alpha}}\right\}=s^{\alpha} L\{f(t)\}
$$

Where $\alpha$ is the fractional order and can be portrayed in the frequency domain by the transfer function $F=\frac{1}{s^{\alpha}}$. [2] Still being the simplest method it can't be directly implemented on electronic circuits thus an effective approach to overcome this issue is by approximating fractional operators by using standard integer order operators. Another method is the approximations of the $1 / \mathrm{s}^{\alpha}$ where $\alpha €(0,1)$ were given in the [09] with errors of approximately $2 \mathrm{~dB}$ for example for the order $\alpha=0.9$ the transfer function will be [9]

$$
\frac{1}{s^{0.9}}=\frac{2.2675(s+215.4)(s+1.292)}{(s+2.154)(s+0.01292)(s+359.4)}
$$

Similarly, linear transfer function approximations were done for fractional order $\alpha$ with maximum discrepancy $3 \mathrm{~dB}$ thus one way to study fractional order chaotic systems isvia linear approximations.

\section{FRACTIONAL ORDER CHAOTIC SYSTEMS}

\section{A. Fractional Lorenz System}

After Lorenz introduced chaos he designed a chaotic system in 1963.The fractional order chaotic Lorenz system will be having the following equations.

$$
\begin{aligned}
& \frac{d^{q} x}{d t^{q}}=\sigma(y-x) \\
& \frac{d^{q} y}{d t^{q}}=r x-x z-y \\
& \frac{d^{q} z}{d t^{q}}=-b z+x y
\end{aligned}
$$

here ' $q$ ' is taken as fractional order and $q €(0,1)$. In the case of Lorenz chaotic system, it was observed that at $q=0.99$ the attractors obtained were better than that of integer order and 0.91 was the fractional order threshold below which suitable chaotic behavior was not obtained thus to obtain best possible attractors via fractional order Lorenz chaotic system the dimension order should be $0.99 * 3=2.97$ (for $\mathrm{x}, \mathrm{y}$ and $\mathrm{z}$ dimensions combined). The effective dimension of $\sum=2.91$ is the threshold of the Lorenz system but it can't be termed as universal threshold for all the systems as each system will behave differently to fractional order [30]. Thus, it was found out that the effective dimension can be a non-integer value and the dynamics of the system depend on it and is highly sensitive to the fractional order. [41]

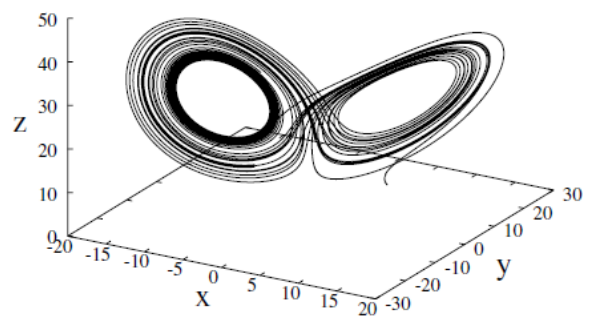

Fig. 2.1 Fractional order Lorenz Chaotic attractor

\section{B. Fractional order Rossler System}

Fractional order Rossler system was researched and it was analyzed numerically using the ADM (Adomian 
Decomposition) method and the further realized on DSP board [31]. ADM method has been used on other chaotic systems (such as Lorenz, Chen, etc.) as it can deal with both linear and non-linear systems in time domain. ADM method was used as it had better accuracy, faster convergence and used far less computer resources hence ADM was used to find and answer to the fractional order chaotic systems rather than Adams-Bashforth-Moulton predictor corrector approach. When compared with integer order chaotic systems $(\mathrm{q}=1)$ the system has unique chaotic behavior and dynamic characteristics such as tangent bifurcations and period-doubling. It was observed that the fractional order threshold for chaotic behavior of the Rossler system was of $\sum=1.119$ (dimension of the system) and $\mathrm{q}=0.346$ when $\mathrm{a}=0.55, \mathrm{~b}=2$ and $\mathrm{c}=4$ thus for $\mathrm{q} \in[0.346,1]$ the system remains in state of chaos. [31]

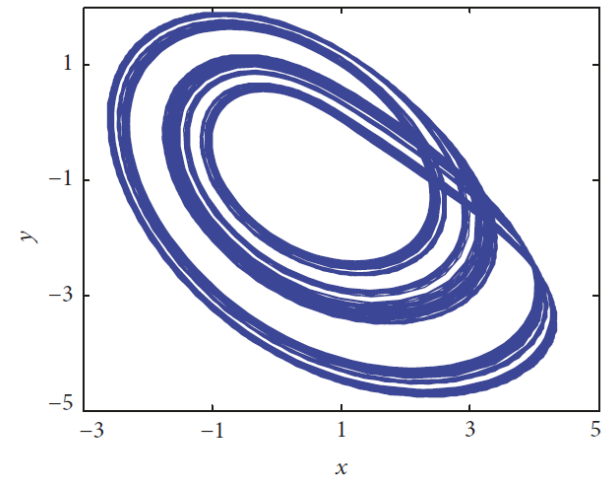

Fig 2.2 Chaotic attractor of Fractional order Rossler system

Following are the differential equations of the system

$$
\begin{gathered}
\frac{\mathrm{d}^{\mathrm{q}} \mathrm{x}}{\mathrm{dt^{ \textrm {q } }}}=-\mathrm{y}-\mathrm{z} \\
\frac{\mathrm{d}^{\mathrm{q}} \mathrm{y}}{\mathrm{dt}^{\mathrm{q}}}=\mathrm{x}+\mathrm{ay} \\
\frac{\mathrm{d}^{\mathrm{q}} \mathrm{z}}{d \mathrm{t}^{\mathrm{q}}}=\mathrm{b}+\mathrm{z}(\mathrm{x}-\mathrm{c})
\end{gathered}
$$

\section{Fractional order Chen System}

Chen chaotic system was studied for fractional order [23] by researchers due to the interesting finding which showed that Chen chaotic system showed chaotic behavior even for $\mathrm{q}=0.3$ which is the least order for which chaotic nature is observed for any system yet. Laplace Transform approach was used to implement fractional order on Chen chaotic system in [33] where the $F=\frac{1}{s^{q}}$ [3-4] transfer function is used in frequency domain as fractional order integrals and differentials don't allow direct implementation in time domain as discussed in [2]. The fractional order chaotic system can be defined with the following differential equations [35]

$$
\begin{aligned}
& \frac{d^{q} x}{d t^{q}}=a(y-x) \\
& \frac{d^{q} y}{d t^{q}}=c y-x z+(c-a) \\
& \frac{d^{q} z}{d t^{q}}=-b z+x y
\end{aligned}
$$

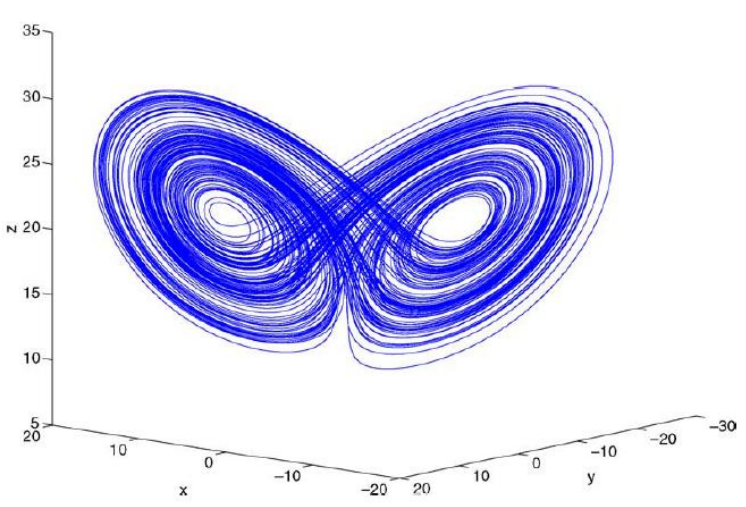

Fig.2.3 Chaotic attractor for Fractional order Chen system at $\mathrm{q}=0.8$

\section{Fractional order Unified chaotic system}

Fractional order circuit implementation of unified chaotic system was studied in [4] at $\mathrm{q}=0.9\left(\sum=2.9\right)$ and for $\mathrm{v}=0.5,0.8$ and 1 the Lyapunov exponents were 0.0214,0.0302 and 0.0223 respectively. Even here the Laplace transform approach was used to design the fractional order system. Using circuit theory in Laplace domain the tree shape model values for $\mathrm{R} \& \mathrm{C}$ were obtained. Hence by replacing the capacitor with this model in the respective integer order system circuit the fractional order implementation was done in [4]. The threshold fractional order to obtain chaos was observed as 2.76 [38] with the fractional order $\mathrm{q} 1=0.93$, $\mathrm{q} 2=0.94$ and $\mathrm{q} 3=0.95$ for $\mathrm{x}, \mathrm{y}$ and $\mathrm{z}$ dimension respectively. The differential equations will be as follows

$$
\begin{gathered}
\frac{d^{q 1} x}{d t^{q}}=(25 \gamma+10)(y-x) \\
\frac{d^{q 2} x}{d t^{q}}=(28-35 \gamma) x-x z+(29 \gamma-1) y \\
\frac{d^{q 3} z}{d t^{q}}=x y-(\gamma+8) z / 3
\end{gathered}
$$



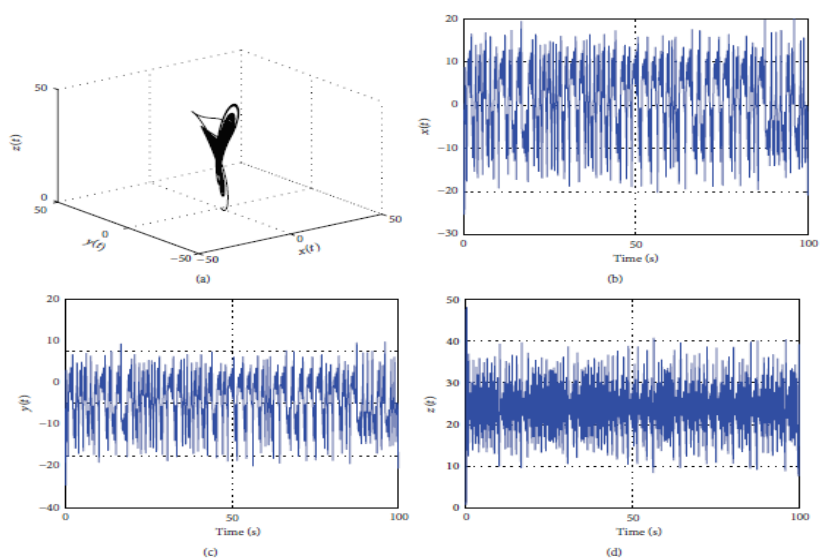

Fig.2.4 Chaotic attractor and time series output of Fractional order Unified chaotic system for $\gamma=0.5$

Chaos is observed in any system when the Lyapunov exponents are greater than zero.

\section{E. Fractional order Lü Chaotic system.}

The fractional order differential equations of the Lü system are [36]

$$
\begin{aligned}
& \frac{\mathrm{d}^{\mathrm{q}} \mathrm{x}}{\mathrm{dt}^{\mathrm{q}}}=\mathrm{a}(\mathrm{y}-x) \\
& \frac{\mathrm{d}^{\mathrm{q}} \mathrm{y}}{\mathrm{dt}^{\mathrm{q}}}=\mathrm{cy}-\mathrm{xz} \\
& \frac{\mathrm{d}^{\mathrm{q}} \mathrm{z}}{\mathrm{dt}^{\mathrm{q}}}=-\mathrm{bz}+\mathrm{xy}
\end{aligned}
$$

here the fractional order is $\mathrm{q}$ and $(\mathrm{a}, \mathrm{b}, \mathrm{c})$ are parameters of the system whose values are $(36,3,20)$ and predefined conditions are $\mathrm{x}=0.2, \mathrm{y}=0.5$ and $\mathrm{z}=0.3$.

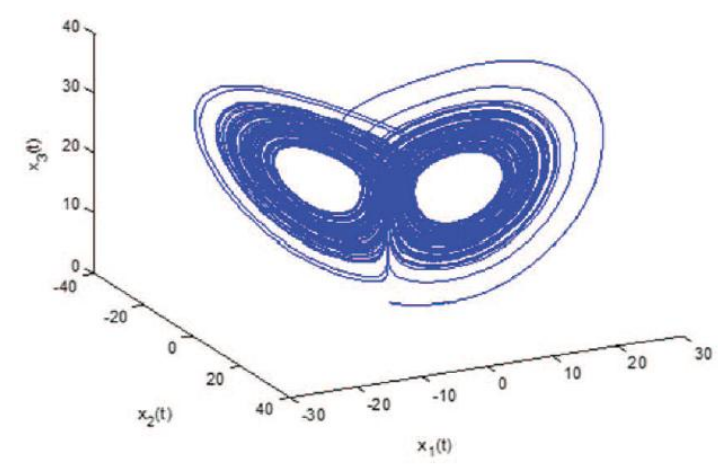

Fig.2.5 Chaotic attractor for Fractional order Lü system for $\mathrm{q}$ $=0.96$

\section{F. Fractional order Pehlivan Chaotic system}

The Pehlivan system was implemented for fractional order in [2] which used the Laplace transform approach as used earlier in unified chaotic system. Here fractional order $\mathrm{q}=0.9$ (2.7 order system) was the fractional order used and attractors, phase portraits, time series and FFT spectrum are compared with its integer order counterpart. The tree shape model was used to replace the capacitors in Pehlivan integer order chaotic circuit and the values of $\mathrm{R} \& \mathrm{C}$ were obtained using circuit theory in Laplace domain where it was seen that the fractional order system had better bandwidth response as compared to integer order and even the attractors were denser than that or integer order chaotic system. The differential equations of this Pehlivan system are

$$
\begin{aligned}
& \frac{\mathrm{d}^{\mathrm{q}} \mathrm{x}}{\mathrm{dt}^{\mathrm{q}}}=a x+b y+y z \\
& \frac{\mathrm{d}^{\mathrm{q}} \mathrm{y}}{\mathrm{dt}^{\mathrm{q}}}=-a y-b x+x z \\
& \frac{\mathrm{d}^{\mathrm{q}} \mathrm{z}}{\mathrm{dt}^{\mathrm{q}}}=\mathrm{z}-\mathrm{xy}
\end{aligned}
$$
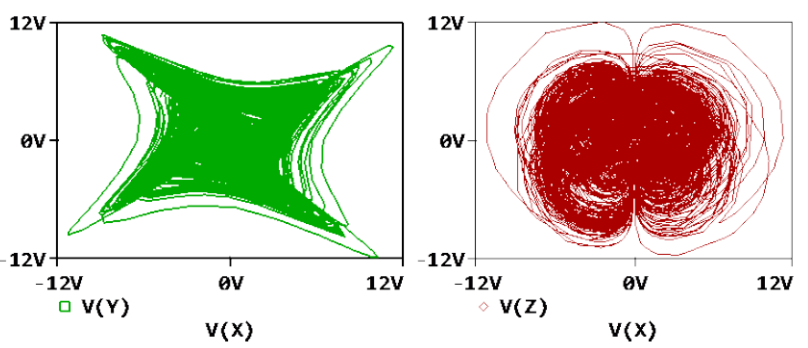

Fig. 2.6 $\mathrm{X}-\mathrm{Y}$ and $\mathrm{X}-\mathrm{Z}$ plane chaotic attractors of fractional order Pehlivan system.

\section{G. Fractional order Chua Chaotic system.}

The fractional order differential equations of the Chua Chaotic systems are [34]

$$
\begin{gathered}
\frac{\mathrm{d}^{\mathrm{q}} \mathrm{x}}{\mathrm{dt}^{\mathrm{q}}}=\mathrm{a}\left(\mathrm{y}+\frac{\mathrm{x}-2 \mathrm{x}^{3}}{7}\right) \\
\frac{\mathrm{d}^{\mathrm{q}} \mathrm{y}}{\mathrm{dt}^{\mathrm{q}}}=\mathrm{x}-\mathrm{y}+\mathrm{z} \\
\frac{\mathrm{d}^{\mathrm{q}} \mathrm{z}}{\mathrm{dt}^{\mathrm{q}}}=-\mathrm{by}
\end{gathered}
$$

The approximations were used from Table 2 for the fractional order and the Laplace transform approach was followed [2-4]. The value of $b$ is found out to be 100/7. The double scroll attractor was visualized as follows 


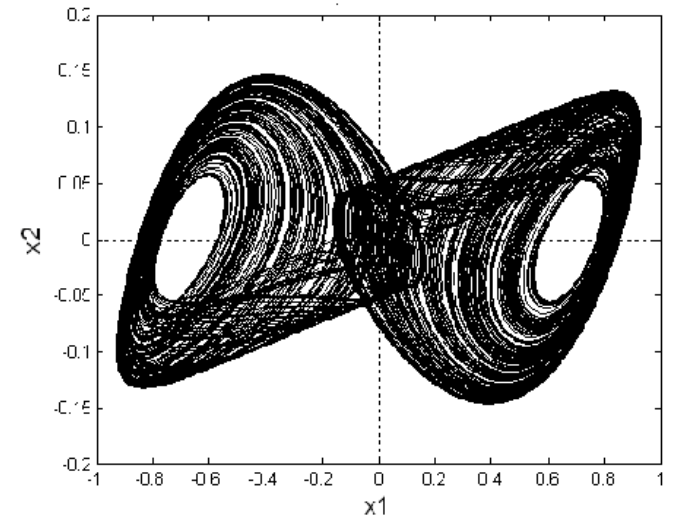

Fig.2.7 Chaotic attractor for Fractional order Chua system.

\section{H. Fractional order Liu Chaotic System}

The fractional order Liu system can be represented as follows

$$
\begin{aligned}
& \frac{d^{q 1} x}{d t^{q}}=\mathrm{a}(\mathrm{y}-\mathrm{x}) \\
& \frac{d^{q 2} x}{d t^{q}}=\mathrm{bx}-\mathrm{lxz} \\
& \frac{d^{q 3} z}{d t^{q}}=\mathrm{cz}+\mathrm{h} x^{2}
\end{aligned}
$$

where $\mathrm{q} 1, \mathrm{q} 2$ and $\mathrm{q} 3$ are fractional orders of $\mathrm{x}, \mathrm{y}$ and $\mathrm{z}$ parameters respectively and has $(0,0,0)$ as an equilibria point. [39]
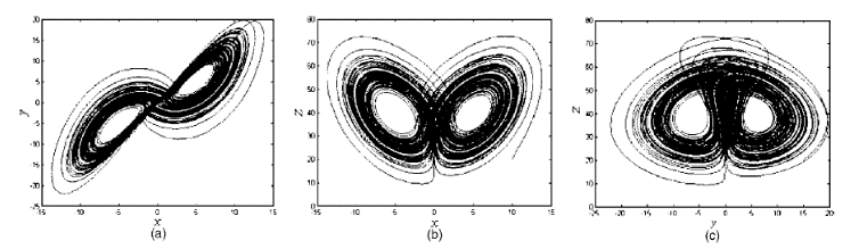

Fig 2.8 Chaotic attractor for Fractional order Liu system at $\mathrm{q} 1=\mathrm{q} 2=\mathrm{q} 3=0.85$

III Synchronization of the Chaotic systems.

Synchronization means when a thing occurs simultaneously or operates in unison [23]. It is a major application of fractional order chaotic system. There are 4 generations of chaotic secure communication systems, the first three generations are based on continuous chaotic synchronization and the fourth generation is based on impulsive methods to improve the bandwidth efficiency than the earlier generations [16]. The first generation is known as chaotic shift keying or additive chaos masking the second generation is known as chaotic modulation the third is called as chaotic cryptosystem. Thus, it was found out that continuous synchronization is less robust than impulsivesynchronization.
Hence, synchronization of the two same fractional order systems or two different fractional order systems or between a fractional order and an integer order system is possible using various synchronization techniques. [43-46]. Synchronization of fractional order Lorenz chaotic system is studied in [41] using master slave method. Synchronization of the fractional order Lorenz and Chen chaotic system is studied and discussed in [44]. The fractional order Chen and Lü system were synchronized by the fractional order sliding mode control method in [23]. The synchronization of fractional order Rossler system was studied in [46] and it was used convert the chaos signal into required signal which is bounded and is also a useful signal. Synchronization of fractional order Liu chaotic systems is carried out in [40] using the adaptive control method and in [39] using linear coupling method. In [34] fractional order Qi and Lü are nonlinearly synchronized and numerical methods were performed to monitor the efficiency of the used synchronized method. Unified chaotic system's synchronization was studied in [44] using impulsive control method and later mathematical simulations are carried out to test the feasibility of the synchronized system. In [43] the flexibility of synchronization was tested as the user could switch between fractional order and integer order depending upon on the desired goal to be achieved. In [45] a new fractional order chaotic system was tested for synchronization. This was Lorenz-Stenflo (LS) system, then using the stability theory the synchronization was carried out with mathematical calculations and simulations for verification.

\section{Applications of chaotic systems}

The major application of chaotic systems are stabilization and control using the extreme sensitivities of the systems as the output of the chaotic systems heavily depend on the changes made in the initial conditions [48]. Synchronization of 2 chaotic signals can be used for encryption by superimposing a message on one of the signals, then on the receiving end only the user with other sequence can decode the message by removing the chaotic masking component.

Chaotic oscillators and controllers can be designed with the help of fractional order chaotic systems [37]. Fractional order systems can be also used for spreading the signal which will in turn cause higher security of the message signal. A signal can be hidden in the noise and be transmitted at low power which increase message privacy between the sender and receiver.

Chaotic systems are used for short term prediction and hence play a major role in predicting diseases, economy, weather, etc. [48]

Neural networks learn by their own just like biological systems hence this approach can be applied for prediction and control such as researchers have already started the enhancement of handwriting recognition using chaotic neural networks. 
Fuzzy logic can also be implemented on various fractional order chaotic systems in the control domain.

Random number generators (RNG) [52] can be designed with the fractional order chaotic systems [22] and using the RNG based encryption algorithms chaos can be implemented in various field such as image processing [50], physics and mechatronics. Basically, fractional order system has similar applications as the integer order chaotic systems, but they are efficient than the integer order systems due to their highly dynamic nature which mimics the real objects or systems in the nature thus, giving the user the choice to choose between them both depending upon the desired purpose. Hence, with the help of chaos we can design systems that are as flexible as the biological systems in the nature, but the advantages should more than enough to counter the complexity introduced due to incorporation of the fractional order systems.

\section{CONCLUSION}

In this review, we have studied and discussed various fractional order chaotic systems. We have studied different chaotic systems with the help of their differential equations discussed how the incorporation of fractional order changes the chaotic behavior of the already highly sensitive nonlinear systems. It was seen that the each chaotic has its own unique chaotic attractor. A small change in the system cause a considerable change in the output of the system.

With this analysis, we can conclude that fractional order improves the dynamic nature of the already sensitive chaotic systems. Different techniques of fractional order implementations were studied and discussed and with the help of these methods oth-er chaotic systems can also be realized and implemented with fractional order.

\section{FUTURE SCOPE}

There are various other chaotic systems which can be implemented with fractional order and can be studied and discussed thus can be incorporated in such reviews.

\section{REFERENCES}

[1] Alpana Pandey, R.K. Baghel, R. P. Singh, "Analysis and Circuit Realization of a New Autonomous Chaotic System, International Journal of Electronics and Communication Engineering ISSN 0974-2166 Volume 5, Number 4 pp. 487-495, 2012.

[2] A. Akgul, B. Aricioglu, S. Kacar, I. Pehlivan. "Circuit Implementations of A Nonlinear System for Integer and Fractional Orders", 8 th International Advanced Technologies Symposium, 2017.

[3] Wajdi M. Ahmad, J.C. Sprott, "Chaos in fractionalorder autonomous nonlinear systems", Chaos, Solitons and Fractals 16 339-351, 2003.
[4] Chen Xiang-Rong, Liu Chong-Xin, and Wang FaQiang, "Circuit realization of the fractional-order unified chaotic system", Chin. Phys. and IOP Publishing Ltd Soc.1674-1056/2008/17(05)/166406 Vol 17 No 5, May 2008

[5] Choksi Hetal and Dr. M. G. Timol, "On the Formulation of Fractional Differential Equation from Differential Equation", International Journal of Dynamics of Fluids. ISSN 0973-1784 Volume 13, Number 2 pp. 209-218, 2017.

[6] YangQuan Chen, Ivo Petr'a s and Dingy"uXue, "Fractional Order Control - A Tutorial", 2009 American Control Conference Hyatt Regency Riverfront, St. Louis, MO, USA June 10-12, 2009.

[7] L'ubomírDorčák, JurajValsa, Emmanuel Gonzalez, JánTerpák, Ivo Petráš and LadislavPivka, "Analogue Realization of Fractional-Order Dynamical Systems",ISSN 1099-4300 www.mdpi.com/journal/entropy, 2013

[8] AlekseiTepljakov, Eduard Petlenkov, Emmanuel A. Gonzalez, Ivo Petras, "Design of a Matlab Design of a Matlab-Based Teaching tool in Introductory Fractional-order systems and controls", The 47TH Annual Frontiers in Education (FIE) Conference, 2017.

[9] Sangeeta Gupta, Smriti Srivastava and Pragya Varshney, "Fractional Order Chaotic Systems: A Survey", International Conference on Computing, Communication and Automation (ICCCA2017)

[10] Dr. Hans J. Haubold," Chapter-9 Applications to Astrophysics Problems", Office of Outer Space Affairs, United Nations, at the 5th SERC School

[11] Ivo Petras and Dagmar Bednarova, "Fractional Order Chaotic Systems", Institute of Control and Informatization of Production Processes BERG Faculty, Technical University of Kosice.

[12] Peter Stavroulakis," Chaos applications in telecommunications", Taylor and Francis Group, 2006

[13] K. Sayevand, A. Golbabai b, Ahmet Yildirim. "Analysis of differential equations of fractional order", Applied Mathematical Modelling 36 43564364, 2012

[14] Edward N. Lorenz, "Deterministic Nonperiodic Flow", Journal of the Atmospheric Sciences, 1963

[15] Jinhu Lu, Guarrong Chen, Daizhan Cheng and SergejCelikovsky, "Bridge the Gap Between the Lorenz system and the Chen System", International Journal of Bifurcation and Chaos Vol. 12, No. 12 29172926,2002

[16] Tao Yang," A Survey of chaotic Secure Communication systems", International Journal of Computational Cognition (http://www.YangSky.com/yangijcc.htm) Volume 2, Number 2, Pages 81-130, June 2004 
[17] Leon 0. Chua, Chai Wah Wu, Anshan Huang, and Guo-Qun Zhong,"A Universal Circuit for Studying and Generating Chaos-Part I: Routes to chaos

[18] ErcanKose, PhD, AzizeAkcayoglu, "Examination of the Eigenvalues Lorenz Chaotic systems", European Scientific Journal /SPECIAL/ edition ISSN: 1857 - 7881 (Print) e - ISSN 1857- 7431, May 2014

[19] Pierre Gaspard,"Rossler systems", Encyclopedia of Nonlinear Science, (Routledge, New York) pp. 808-811, 2005

[20] K M Ibrahim, K., R K Jamal, \& F.H Ali, "Chaotic behaviour of the Rossler model and its analysis by using bifurcations of limit cycles and chaotic attractors.", Journal of Physics: Conference Series. 1003. Institute of Physics Publishing, ,2018.

[21] S. Larptwee and W. San-Um, "Implementation of Rössler Chaotic System through Inherent Exponential Nonlinearity of a Diode with TwoChannel Chaotic

Synchronization Applications", 2013 Fourth International Conference on Intelligent Control and Information Processing (ICICIP), Beijing, China, June 9 - 11, 2013

[22] Monika Priya, Girada Swetha, Ramji Gupta, AlpanaPandey,"Compact Chaotic Oscillator Using $180 \mathrm{~nm}$ CMOS technology for its use in True Random Number Generator", Proceeding International conference on Recent Innovations is Signal Processing and Embedded Systems (RISE2017) 27-29 October,2017

[23] Yong $\mathrm{Xu}$ and Hua Wang, "Synchronization of Fractional-Order Chaotic Systems with Gaussian Fluctuation by Sliding Mode Control," Abstract and Applied Analysis, vol. 2013, Article ID 948782, 7 pages, 2013.

[24] Petráš, I. "A note on the fractional-order Chua's system.", Chaos, Solitons and Fractals, 38(1), 140147, 2008.

[25] Mingjun Wang, "Chaotic Control of Lü System via Three Methods", International Journal of Modern Nonlinear Theory and Application, 2014, 3, 29-36, June 2014

[26] Lj. M. Kocic, S.Gegovska-Zajkova, S. Kostadinova,"On Chua Dynamical System", Scientific Publications of the Statue University Of Novi Pazar, vol. 2, 1 53-60, 2010,

[27] Ihsan Pehlivan, YılmazUyaro Glu. "A new chaotic attractor from general Lorenz system family and its electronic experimental implementation", Turk J Elec Eng\& Comp Sci, Vol.18, No.2, 2010.

[28] Guanrong Chen, "The Chen system revisited", Dynamics of Continuous, Discrete and Impulsive Systems Series B: Applications \& Algorithms 20 691-696, 2013.
[29] Liangqiang Zhou and Fangqi Chen, "Sil'nikov chaos of the Liu system", American Institute of Physics., Chaos 18, $013113,2008$.

[30] Ilia Grigorenko and Elena Grigorenko, "Chaotic Dynamics of the Fractional Lorenz System", Physical Review letters Vol.91, No.3, 18-July-2003

[31] Huihai Wang, Shaobo $\mathrm{He}$, and Kehui Sun, "Complex Dynamics of the Fractional-Order Rössler System and Its Tracking Synchronization Control," Complexity, vol. 2018, Article ID 4019749, 13 pages, 2018

[32] Akif Akgul, Irene Moroz, Ihsan Pehlivan, SundarapandianVaidyanathana," A new four-scroll chaotic attractor and its engineering applications", Optik - Int. J. Light Electron Opt, 2016,

[33] Jun Guo Lu, Guanrong Chen, "A note on the fractional-order Chen system", Chaos, Solitons and Fractals 27 685-688, 2006.

[34] Tom T.Harley, Carl F Lorenzo, and Helen KilloryQammar,"Chaos ins fractional order Chua system", NASA Technical paper 3543, January 1996.

[35] Chunguang Li, Guanrong Chen, "Chaos in the fractional order Chen system and its control", Chaos, Solitons and Fractals 22 549-554, 2004.

[36] Vijay K. Yadava, Subir Das, and Donato Cafagna" Nonlinear synchronization of fractional-order $\mathrm{Lu}$ and Qi chaotic systems" IEEE International Conference on Electronics, Circuits and Systems (ICECS), 2016

[37] Girada Swetha, Monika Priya, Ramji Gupta and Dr.Alpana Pandey, "Implementation of chaotic oscillator using Matlab", Proceeding International conference on Recent Innovations is Signal Processing and Embedded Systems (RISE-2017) 27-29 October,2017.

[38] Jian Yuan, Bao Shi, Xiaoyun Zeng, Wenqiang Ji, and Tetie Pan, "Sliding Mode Control of the Fractional-Order Unified Chaotic System," Abstract and Applied Analysis, vol. 2013, Article ID 397504, 13 pages, 2013

[39] Xing-yuan Wang and Ming-jun Wang," Dynamic analysis of the fractional-order Liu system and its synchronization", American Institute of Physics. Chaos 17, 033106, 2007

[40] AdelehNourian and Saeed Balochian," The adaptive synchronization of fractional-order Liu chaotic system with unknown parameters", PramanKournalof Physics, Indian Academy of Sciences Vol. 86, No.6 physics pp. 1401-1407, June 2016

[41] Sachin Bhalekar," Chaos Control and Synchronization in Fractional-Order Lorenz-Like System", International Journal of Differential Equations Volume 2012, Article ID 623234, June 2012 
[42] Chunguang Lia and Guanrong Chen," Chaos and hyperchaos in the fractional-order Rossler equations", Physica A $34155-61,2004$

[43] Manashita Borah, and Binoy K. Roy," Switching Synchronisation Control between Integer-order and Fractional-order Dynamics of a Chaotic System", 2017 Indian Control Conference (ICC) Indian Institute of Technology, Guwahati, India, January 4-6, 2017.

[44] Zhou Ping, Cheng Yuan-Ming, and Kuang Fei, "Synchronization between fractional-order chaotic systems and integer orders chaotic systems (fractional-order chaotic systems), Chin. Phys. B Vol. 19, No. 9 090503, 2010.

[45] Liang Shan, Zhong Liu, Jun Li, and Zhiquan Wang, "A New Fractional-order Chaotic System and its Synchronization Control", Joint 48th IEEE Conference on Decision and Control and 28th Chinese Control Conference Shanghai, P.R. China, December 16-18, 2009.

[46] H.N. Agiza and M.T. Yassen,"Synchronization of Rossler and Chen chaotic dynamical systems using active control", Physics Letters A 278 191-197, January-2001

[47] Xu, G.; Shekofteh, Y.; Akgül, A.; Li, C.; Panahi, S. A New Chaotic System with a Self-Excited Attractor: Entropy Measurement, Signal Encryption, and Parameter Estimation. Entropy 2018

[48] William Ditto and ToshinoriMunakata, "Principles and Applications of Chaotic Systems", Communications of the ACM, Vol38, No.11, November 1995

[49] Abdolmohammadi, Hamid Reza \& Khalaf, Abdul Jalil M. \& Panahi, Shirin \& Rajagopal, Karthikeyan \& Pham, Viet-Thanh \& Jafari, Sajad, "A new 4D chaotic system with hidden attractor and its engineering applications: analog circuit design and FPGA implementation". Pramana. 90. 10.1007/s12043-018-1569-2. ,2018

[50] Adel Ouannasa, Ahmad Taher Azarb* $\dagger$ and SundarapandianVaidyanathanc,"A robustmethod for new fractional hybrid chaos synchronization", Published online in Wiley Online Library Math. Meth. Appl. Sci., 40 1804-1812, 2017.

[51] SezginKac, ar, Analog Circuit and Microcontroller Based RNG Application of a New Easy Realizable 4D Chaotic System, Optik - International Journal for Light and Electron Optics, 2016 